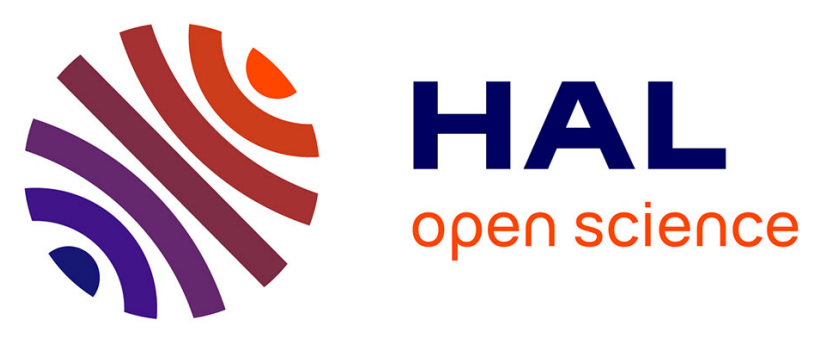

\title{
Une sociologie critique des usages est-elle possible? Réflexions sur la conciliation d'une posture critique et d'une démarche empirique
}

\author{
Alexandre Coutant, Jean-Claude Domenget
}

\section{To cite this version:}

Alexandre Coutant, Jean-Claude Domenget. Une sociologie critique des usages est-elle possible? Réflexions sur la conciliation d'une posture critique et d'une démarche empirique. Communication \& organisation: perspectives critiques, Presses du Septentrion, pp.143-154, 2013, 978-2757404300. hal-01187854

\section{HAL Id: hal-01187854 \\ https://hal.science/hal-01187854}

Submitted on 27 Aug 2015

HAL is a multi-disciplinary open access archive for the deposit and dissemination of scientific research documents, whether they are published or not. The documents may come from teaching and research institutions in France or abroad, or from public or private research centers.
L'archive ouverte pluridisciplinaire HAL, est destinée au dépôt et à la diffusion de documents scientifiques de niveau recherche, publiés ou non, émanant des établissements d'enseignement et de recherche français ou étrangers, des laboratoires publics ou privés. 


\title{
Une sociologie critique des usages est-elle possible ? Réflexions sur la conciliation d'une posture critique et d'une démarche empirique ${ }^{1}$
}

\author{
Alexandre COUTANT et Jean-Claude DOMENGET ${ }^{2}$
}

Résumé : l'analyse critique d'un état actuel de l'inscription d'une technologie dans la société est difficile puisque les imaginaires associés aux TNIC ne peuvent être facilement renvoyés à des résultats stabilisés concernant les usages. Les conditions d'une analyse sociotechnique de tels phénomènes sont évoquées dans le but de réconcilier des approches critiques et empiriques en sociologie des usages. Mots-clés : analyse critique, sociologie des usages, TNIC

\section{L'idéologie des technologies numériques de l'information et de la communication}

Les technologies numériques de l'information et de la communication (TNIC) charrient un imaginaire social que le chercheur ne peut ignorer (Flichy, 2001). Cette faculté à engendrer l'idéologie a régulièrement mené les commentateurs des TNIC à tenir des positions radicalement opposées, que l'on a pris coutume d'opposer en sociologisme ou technicisme. Ces postures ont contribué à occulter d'autres approches proprement sociotechniques, abordant tout à la fois la société, l'individu et les objets dans un processus de co-construction permanente (Akrich, 1994).

Les évolutions du web regroupées sous l'appellation aussi englobante que floue de web 2.0 n'échappent pas à cette tendance (Rebillard, 2007). L'inflation des termes quasi-synonymes, web social/participatif/communautaire, réseau social/communautaire, médias sociaux, est un bon indicateur de la vigueur de l'idéologie (Stenger et Coutant, 2011a). Replacer ces discours dans la conjoncture socio-économique de la phase de développement d'internet dans les années 2000 permet alors de comprendre le rôle promotionnel de la «révolution participative » (O'Reilly, 2005). Ces discours apparaissent dans le contexte d'une nécessité de redynamiser l'engouement pour la nouvelle économie (Bouquillion et Matthews, 2010). L'analyse des derniers discours promotionnels des «gourous » de l'internet révèle d'ailleurs ce passage d'un imaginaire de la participation à une stratégie bien moins émancipatrice d'exploitation de celle-ci (Rebillard, 2011). L'attention aux dimensions culturelles, marchandes et techniques (Kline et al., 2003) s'avère ici fondamentale pour éviter de réifier l'une de celles-ci.

\footnotetext{
${ }^{1}$ Ce travail est issu du projet de recherche IDENTIC, lauréat de l'appel à projets « Web innovant » financé par le secrétariat d'État chargé de la prospective et du développement de l'économie numérique. Il réunit le Groupe La Poste, la société My.id.is et le laboratoire CEREGE (Université de Poitiers). Nous voulons ici les remercier ainsi que les collègues des laboratoires partenaires.

${ }^{2}$ Université de Franche-Comté, Elliadd, coutant.alexandre@gmail.com ; jcdomenget@gmail.com
} 
Cependant, la plupart de ces travaux repose sur une approche historique comparative permettant d'aborder avec davantage de recul le potentiel que recèlent les nouvelles TNIC. Ainsi, l'aspect le plus prosaïque et quotidien de l'appropriation des outils et services qu'elles proposent est peu mis en avant (Granjon, 2004). Les particularités des configurations sociotechniques émergentes risquent alors d'être ignorées.

Pour parer à cette méconnaissance, la sociologie des usages s'est proposée d'investiguer les pratiques contemporaines des usagers des TNIC tout en conservant un point de vue critique (Vitalis, 1994 ; Granjon, 2004 ; Proulx, 2005), mais les régulières synthèses des travaux effectués dans ce courant témoignent de la difficulté de réellement tenir ce rôle (Chambat, 1994 ; Jouët, 2000 ; Denouël et Granjon, 2011).

Nous souhaitons revenir dans cet article sur la complexité d'une analyse des phénomènes d'appropriation de TNIC en cours de structuration. Une fois évoqués les modèles proposés par la sociologie des usages et leurs limites pointées par ses contributeurs, nous présenterons les terrains sur lesquels nous avons tenté de mettre en place une telle analyse. Nous pourrons envisager alors des pistes de discussion qui amèneraient à une réconciliation des approches critiques et empiriques en sociologie des usages.

\section{Quelle posture critique pour la sociologie des usages ?}

\section{Un projet ambitieux}

Une dimension critique dans la sociologie des usages se justifie par l'importance de disposer d'un «garde fou intellectuel» face à la force des idéologies associées aux TNIC. Cette attention critique relève de différents niveaux. Tout d'abord le rappel de la complexité du phénomène : si la technique est bien un « rapport social matérialisé » (Roqueplo, 1983, p. 162), il convient d'en tirer toutes les conclusions concernant la prise en compte, au sein du phénomène, des différentes dimensions sociales, économiques et technologiques. L'attention au lexique employé ensuite : celui-ci est le premier porteur des discours promotionnels, comme le démontrent actuellement les termes si ambivalents d'« amis », «curateurs », «participation», «partage», etc. (Coutant et Domenget, 2011). Le recul historique enfin, qui permet de comparer l'inscription sociale des TNIC à celle de leurs ancêtres, et de prendre la mesure de l'écart entre la vision des concepteurs et l'actualisation de leurs projets, ainsi que de la permanence de certaines attentes communicationnelles, au-delà des « révolutions » promues avec chaque innovation (Perriault, 2009).

La sociologie des usages a aussi clairement cherché à se démarquer de ce qu'elle a identifié comme une «demande sociale» (Jouët, 2000). Bien que le débat soit difficile à conclure sur la position d'extériorité ou participative que doit adopter le chercheur face aux projets d'innovation quand ceux-ci soulèvent des enjeux sociaux, politiques, parfois même anthropologiques, force est de constater que cette question se pose d'autant plus fortement aujourd'hui que les financements sur contrat proposés aux chercheurs valorisent les conglomérats mixtes. Face à ces enjeux, la position critique, développée 
par la sociologie des usages, consiste en une mise à distance du discours spontané ou promotionnel, reposant sur une «manière de poser des problèmes et de construire des théories » (Kaufmann, 2006, p. 20). Plus largement, l'approche critique de la sociologie des usages conduit à proposer un cadre d'analyse ambitieux qui doit permettre d'éviter un double écueil: celui d'une approche communicationnelle (Jouët, 2000), qui confond communication et social et celui de l'économie interne des usages (Jauréguiberry, 2008), «naturalisant » les usages par une analyse certes fine mais restant collée à cette ethnographie minutieuse. Cette ambition a été d'abord au cœur du programme de la socio-politique des usages, appelant à ne pas réduire l'explication des usages à un principe unique mais plutôt à prendre constamment en compte «les interrelations complexes entre outil et contexte, offre et utilisation, technique et social $\gg$ (Vedel dans Vitalis, 1994, p.32). Le nécessaire élargissement des problématiques à une approche critique a ensuite abouti à une théorie des usages (Proulx, 2001, 2005), c'est-à-dire un modèle de « construction sociale des usages », articulant dimension descriptive et dimension normative. Enfin, il s'agit d'être attentif à la coproduction des rapports sociaux et des rapports de sens, autrement dit d'interroger systématiquement le sens que les usages de la technique prennent pour les utilisateurs et tenter de montrer ce que cela dévoile de leur existence sociale (Denouël et Granjon, 2011).

\section{Une application exhaustive difficile}

Ces ambitions qui ancrent la sociologie des usages dans une approche clairement interdisciplinaire sont souvent restées programmatiques. La question de comment arriver à respecter tous les engagements de cette démarche exigeante reste posée.

Si nous avons insisté sur la nécessité de dénoncer les discours technicistes et promotionnels ne manquant pas d'accompagner toute technologie, nous devons aussi constater que ces derniers se retrouvent bien plus régulièrement que les travaux observant les usages. Reposant sur des analyses des discours accompagnant les TNIC, ils ont pour conséquence de laisser finalement peu de visibilité aux pratiques effectives des utilisateurs. Cette attitude a été d'autant plus encouragée que l'empirisme a rapidement été perçu comme la position à combattre par les sociologues des usages, qui qualifient régulièrement son développement de «menace» (Chambat, 1994, p. 263 ; Jouët, 2000, p. 491 et dès le résumé de l'article). Signalons ici une divergence avec la théorie critique classique qui a rapidement fait reposer sa démarche sur des travaux de terrains ${ }^{3}$.

Les intérêts et limites de la sociologie des usages se révèlent d'une manière particulière lorsque le chercheur prend pour objet des TNIC en cours d'appropriation, donc des phénomènes encore non stabilisés. Il est en effet alors difficile de prédire ce qui va réellement s'actualiser dans le potentiel des innovations, ce qui rend d'autant plus nécessaire l'attention à la complexité des manières qu'ont les individus de développer des usages de ces dernières. Un travail de comparaison diachronique risque

\footnotetext{
${ }^{3}$ Entretien avec Voirol : http://www.nonfiction.fr/articleprint-1985-_redecouvrir_la_theorie_critique.htm
} 
alors d'encourager à privilégier la constance de certaines logiques communicationnelles ou de liens sociaux au détriment de la compréhension de la particularité de la configuration sociotechnique (Rebillard, 2007) se constituant. La critique peut dans ce cas porter sur l'idéologie techniciste mais risque les mésinterprétations dès lors qu'elle cherche à se positionner sur les enjeux sociaux et éthiques associés à ces innovations.

La conscience de ces limites nous a conduits à tenter de mettre en place une approche associant position critique et analyse fine des phénomènes d'appropriation.

\section{Les enseignements des terrains}

Le premier terrain concerne les activités des jeunes sur les réseaux socionumériques (Rsn), commandité par le Groupe La Poste. L'approche critique telle que définie supra s'est révélée nécessaire dès le titre de l'appel à projet qui associait comme synonymes Rsn et communautés virtuelles. La demande résultait par ailleurs d'une volonté de comprendre les activités des jeunes sur ces espaces et d'envisager si une présence des marques était tolérable, souhaitable, attendue ou pas. Celle-ci résultait d'une veille des services dédiés à l'innovation, reposant essentiellement sur les discours des consultants en «nouveaux médias ». Ces premiers risques concernant le cadrage du projet de recherche, aporie définitionnelle et problématique orientée par des discours commerciaux, ont nécessité d'y consacrer la première partie de l'enquête. D'une part, comme nous l'avons souligné, il a été nécessaire de résoudre les difficultés dues à l'inflation des termes qualifiant les formes pourtant particulières de groupes sociaux se retrouvant sur ces espaces. En effet, le type de lien se formant entre les utilisateurs s'écarte des définitions classiques des communautés et appelle à une appréciation critique de ce qui est entendu par amitié. Cependant, rappeler que le fait que Facebook qualifie ses mises en relation d' «amitié » ne suffit pas à transformer nos contacts en réels amis n'a pu constituer qu'une première étape, amenant dans un second temps à investiguer de manière compréhensive les formes relativement inédites de lien ainsi établis ${ }^{4}$ ainsi que les manières des utilisateurs de se comporter en audiences passives ou en interactants (Stenger et Coutant, 2011b). D'autre part, la déconstruction du discours promotionnel accompagnant les Rsn a permis de reproblématiser les évidences qu'il établissait: nécessité pour les marques d'êtres présentes, mutation radicale de la relation marque/consommateur, intérêt de cet espace pour initier des interactions marchandes, etc. Démontrer l'aspect intéressé de ces discours n'a cependant permis que de rappeler que ces points devaient être interrogés et non pas de fournir des réponses sur le rapport des utilisateurs de ces services aux marques. Cette démarche a par conséquent dû être complétée par un minutieux travail de terrain qui a permis de souligner combien les utilisateurs ne perçoivent pas ces sites comme des espaces marchands ou comment leurs manières de se mettre en relation avec des dispositifs de marques ne constituent ni des preuves d'engagement, ni même nécessairement une attitude positive envers la

\footnotetext{
${ }^{4}$ Couvrant une focale étonnamment large pour un même dispositif puisque cela va de l'entretien de liens faibles à l'ajout d'un vecteur supplémentaire de communication aux relations intenses déjà multisupports.
} 
marque (Coutant et Domenget, 2011). Ajoutons que ce travail a nécessité la convocation de champs de connaissance différents allant de la sociologie de la jeunesse aux recherches en marketing, en passant par la sociologie des réseaux sociaux ou de l'innovation.

À l'opposé, la production empirique disponible au lancement du projet se décomposait essentiellement en travaux menés en Amérique du Nord avec une volonté plus ou moins assumée de coller au point de vue des utilisateurs (Boyd, 2007 et de nombreux articles publiés dans le Journal of Computer Mediated Communication) ou en travaux réinvestissant les théories structurales des réseaux sociaux et le capital social (Lewis et al., 2008 ; Notley, 2009). Deux tendances de ces travaux ont pu être mises en doute. La première consiste à réinvestir les théories du capital social ou de l'analyse structurale des réseaux sociaux, sans examen du sens qu'elles prennent dans le contexte en ligne. La seconde consiste à se focaliser sur l'espace générationnel autonome ainsi rendu accessible aux adolescents, sans accorder d'importance aux logiques commerciales des sites ou à l'influence du système mis en place. L'invitation à une montée en théorie ainsi qu'à la distance à l'égard des discours des utilisateurs a cette fois permis de ne pas se cantonner à une lecture superficielle du phénomène.

La compréhension fine des Rsn n'a été rendue possible que par une méthode de travail consistant à effectuer des allers-retours entre le cadrage critique du phénomène et l'appel à un ensemble varié de protocoles de recueil de matériaux (veille, observations en ligne, entretiens, analyse de profils, questionnaire, analyse logicielle sémantique et structurale).

Le second terrain concerne l'impératif de visibilité pour exister (Aubert et Haroche, 2011), dans notre société actuelle, analysé à partir des usages de Twitter chez des professionnels du web (Domenget, 2012).

L'approche critique a permis d'orienter le questionnement vers des enjeux sociaux souvent oubliés, éthiques notamment, et d'analyser la visibilité comme « une pratique sociale réglée par des normes » (Voirol, 2005). Il s'agit d'interroger la forme de visibilité en jeu, en comparaison de celles précédemment distinguées, parmi lesquelles la visibilité médiatisée, analysée sous l'angle des médiations techniques et des supports symboliques pour augmenter le spectre de «ce qui peut être $\mathrm{vu} »$. L'approche critique nous a conduit ensuite à clarifier le concept de visibilité en abordant deux concepts proches sémantiquement: la reconnaissance et la présence numérique. De nos jours, il semblerait que la reconnaissance ait acquis le statut de valeur (évaluée positivement) alors que la visibilité serait devenue une anti-valeur (évaluée négativement) (Heinich dans Aubert et Haroche, 2011). En fait, ce sont deux phases d'une relation définie par la présence ou non d'une réciprocité et d'une attention mutuelle (Honneth 2004). Avec le concept de présence numérique, Merzeau (2010) propose de s'intéresser aux images, aux traces, aux profils développés par les usagers. La visibilité relève alors de l'ordre de la manifestation (qui peut être rapprochée du régime de l'alerte) et de l'indexation (proche de celui de la fidélisation). Autrement dit, il s'agit d'analyser dans la durée la production continue et illimitée de soi ; l'individu étant jugé, au travers de la quantité de signes, de textes et d'images qu'il produit. 
L'approche critique nous a enfin amenés à distinguer les formats de visibilité (Cardon, 2008), spécifiques à chaque plateforme, et en partant de l'approche sociotechnique proposée par Stenger et Coutant (2011a) qui cartographie les «médias sociaux » entre activités et visibilité, à caractériser Twitter.

Ce travail en amont a été associé à une approche méthodologique privilégiant le terrain, consistant en un recueil pluriel de matériaux ${ }^{5}$. Un va-et-vient a été réalisé entre terrain et théorie lors de la phase d'entretiens, afin de proposer des catégories à visée théorique. Signalons néanmoins que cette remontée en théorie n'est pas possible systématiquement et se révèle extrêmement chronophage.

Les résultats de cette recherche ont permis de poser les bases d'une éthique des " médias sociaux », à travers le cas de Twitter. Explicitement évoquée par certains usagers sous l'angle de la nétiquette de 1995, une telle éthique ne doit pas être confondue avec ces règles de bonnes conduites, ces chartes qui restent «des éthiques sans éthique » (Giffard, 2005). Les enjeux éthiques relèvent d'au moins trois niveaux d'analyse: 1) du côté de la personne avec la protection de la vie privée ; 2) du côté de l'impact sur l'humain en ce qui concerne la lutte contre l'addiction et contre la saturation cognitive $(\mathrm{COS}) ; 3)$ au niveau de la société en rapport avec la qualité des informations. Concevoir une éthique des «médias sociaux» passe par la prise en compte de la diversité des usages dans ces espaces, rassemblant des traditions de professions (journaliste, documentaliste, communiquant, marketeur etc.) ayant des éthiques propres, sans oublier qu'il s'agit d'une éthique en pratique, s'opérant à un niveau individuel, sans forcément ambition d'une dimension collective.

\section{Quelques pistes de réflexion sur une approche critico-empirique}

Ces deux projets encouragent à plaider en faveur d'une démarche critico-empirique pour la sociologie des usages. Les termes critique et empirique ayant coutume d'être opposés en sociologie des usages, nous indiquerons ici plusieurs pistes qui rendraient possible leur réconciliation.

La première consiste dans l'objet particulier de la sociologie des usages, qui la conduit à s'intéresser à des phénomènes en cours de stabilisation. Il parait alors d'autant plus essentiel de connaître précisément le dispositif et les usages qui en sont faits que les deux évoluent vite et s'influencent mutuellement. Négliger cette connaissance fine peut mener à deux risques : la focalisation sur les logiques sociales préexistantes au point d'en occulter ce que modifie la configuration sociotechnique particulière ; l'inaudibilité d'une critique s'en tenant à une position de principe, faute de prise en compte par cette dernière des motivations, pourtant exceptionnellement fortes, à participer. C'est cette complémentarité qui permet de comprendre comment les désormais nombreuses craintes à l'égard de la vie privée ne se traduisent pas par une désaffection de Facebook. Cet apparent paradoxe ne prend effectivement sens qu'une fois bien repéré un ensemble complexe de déterminants des pratiques,

\footnotetext{
${ }^{5}$ Pratiques de l'outil pendant trois ans en immersion, série d'entretiens compréhensifs, complétés par une revue des blogs, livres blancs, études universitaires et professionnelles sur Twitter durant six mois, rédaction de mémos à visée de théorisation.
} 
parmi lesquels un large phénomène de déplacement des frontières de l'intime (Berrebi-Hofmann, 2009); le caractère inédit de l'archivage de traces d'activité usuellement fugaces et inconscientes (Hoog, 2009) ou encore la mise en place d'un cadre d'interaction encourageant ces confusions (Coutant, 2011). La même démarche pourra être suivie pour saisir l'exemple évoqué supra de l'indifférence aux marques dans un espace pourtant excessivement marketé (Stenger, 2011).

La seconde piste de réconciliation consiste à reconnaître que les programmes avancés d'une sociologie critique des usages des TNIC sont peut-être trop ambitieux. Une solution est de multiplier les terrains, en faisant attention à ne pas vouloir redécouvrir les acquis de la sociologie des usages mais plutôt à monter progressivement en théorie par une démarche comparative et à visée macrosociologique. En effet, après trente ans de recherche en sociologie des usages, une question reste posée. Les programmes d'une sociologie critique des usages des TNIC sont-ils réalisables ? Autrement dit, pour reprendre une des interrogations faisant partie du programme de Proulx, «peut-on [réellement] problématiser la question microsociologique des usages et des représentations des objets techniques dans un cadre macrosociologique plus vaste (matrice culturelle et contexte socio-politique) mais en évitant de recourir à la dichotomie stérile macro / micro ?»(Proulx, 2001, p.58). Les ambitions pourtant très stimulantes intellectuellement des différents programmes sont souvent restées lettremorte, que ce soit au niveau de la socio-politique des usages ou d'une théorie des usages. S'il demeure des programmes de recherche visant à articuler les conduites microsociales et les orientations macrosociales (Denouël et Granjon, 2011), il semble bien qu'aujourd'hui le clivage soit établi. D'un côté on assiste au morcellement de travaux, renforcé au sein des internet studies autour de multiples objets et services techniques, accumulant les approches microsociologiques sans ambition de théorisation poussée. Elles ont conduit à une connaissance fine des pratiques spécifiques mais laissent entier le questionnement global par rapport au sens social et aux mutations de la société (Jouët dans Denouël et Granjon, 2011). De l'autre, les approches critiques se sont raréfiées et restent à un niveau macrosociologique sans passage systématique par des terrains. Deux fronts sont évoqués par Jouët : le « nouvel esprit du capitalisme » qui insuffle le développement d'internet (Georges et Granjon, 2008) et les discours promotionnels de la participation marquant les usages du web 2.0 (Rebillard, 2007, 2011). Ajoutons que ces problématiques demeurent très sociologiques et, si elles les évoquent souvent, accordent rarement une attention équivalente aux dimensions économiques (commerciales, industrielles, managériales) ou techniques (affordances des terminaux et logiciels). Pour sortir de cette ornière et monter en théorie dans des temps de recherche finis, il est indispensable de travailler en équipe, dans une optique réellement interdisciplinaire. Ce fonctionnement parait le seul susceptible de permettre de traiter simultanément les multiples dimensions économiques, politiques, sociétales, technologiques liées aux mutations en cours de la société et le rôle que les TNIC y jouent. Dans le cas contraire, les délais s'allongent très fortement et rendent hypothétiques la production de résultats d'un autre niveau que ceux micro, issus du terrain. 
Une exigence non négligeable tient aussi à la nécessité pour le chercheur d'accepter une certaine mobilité théorique. Il devient effectivement difficile pour ce dernier de s'en tenir à son cadre habituel. Les discours de promotion comme les pratiques orientent effectivement vers des traditions de recherche qu'il est nécessaire de s'approprier, même (surtout) lorsqu'il s'agira au final de critiquer leur emploi. Ainsi par exemple de l'analyse structurale des réseaux sociaux, dont le vocabulaire innerve le dispositif des Rsn et les discours des commentateurs, bien que ces outils et théories demeurent difficiles à appliquer en l'état à des plateformes abritant des activités très différentes de celles sur lesquelles ce courant se penche usuellement.

Cette contrainte plaide à nouveau en faveur d'une démarche par équipes interdisciplinaires. Une proposition qui risque d'apporter plus de difficultés que de solutions tant une telle démarche demeure difficile à mettre en place. Il ne s'agit aucunement de sous-estimer cette complexité, qui impose une réflexion proprement épistémologique sur les conditions de l'émergence d'une réelle complémentarité entre disciplines et non d'une simple juxtaposition (Coutant, 2010).

\section{Bibliographie}

Akrich M. 1994. Comment sortir de la dichotomie technique / société, in Latour B., Lemonnier P., De la préhistoire aux missiles balistiques : l'intelligence sociale des techniques, La Découverte, pp. 105131.

Aubert N. et Haroche C. (éditeurs.) 2011. Les tyrannies de la visibilité. Etre visible pour exister?, Eres.

Berrebi-Hoffmann I. 2009. Politiques de l'intime - Des utopies sociales d'hier aux mondes du travail d'aujourd'hui, La Découverte.

Bouquillion P. et Matthews, J-T. 2010. Le web collaboratif : Mutations des industries de la culture et de la communication, $\mathrm{PUG}$.

Boyd D. 2007. Why Youth (Heart) Social Network Sites: The Role of Networked Publics. In Teenage Social Life, MacArthur Foundation Series on Digital Learning - Youth, Identity, and Digital Media Volume, MA: MIT Press.

Cardon D. 2008. Le design de la visibilité : essai de cartographie du web 2.0, Réseaux, nº 152 , pp. 93 137.

Certeau (de) M. 1980. L'invention du quotidien, tome 1 : arts de faire, Gallimard.

Chambat P. 1994. Usages des technologies de l'information et de la communication (TIC) : évolution des problématiques, Technologies de l'information et société, 6 (3), pp. 249-269.

Chatriot A., Chessel M-E., Hilton, M. 2005. Au nom du consommateur : Consommation et politique en Europe et aux États-Unis au XXe siècle, La Découverte.

Coutant A. 2010. Les réseaux socionumériques : un objet de recherche propice à l'interdisciplinarité, 17ème Congrès de la Société Française des Sciences de l'Information et de la Communication, 23-26 
juin, Dijon (France), [ version électronique ] http://tabarqa.ubourgogne.fr/outils/OconfS/index.php/SIC/SFSIC17/paper/view/94/52, le 16 décembre 2011.

Coutant A. 2011. Les réseaux socionumériques, des supports identitaires ambivalents, Hermès, $\mathrm{n}^{\circ}$ 59, pp. 53-58.

Coutant A. et Domenget J-C. 2011. Le web participatif a-t-il besoin des marketeurs ?, 79ème congrès international ACFAS «web social, communautés virtuelles et consommation », 11 mai, Sherbrooke (Canada), [ version électronique ] http://www.crpcm.uqam.ca/pages/docs/centres/cmark/ACFAS_428_Actes_ComMkg_2011.pdf, le 16 décembre 2011.

Coutant A. et Stenger T. 2011. Les activités quotidiennes des jeunes sur les réseaux socionumériques : typologie et enjeux, Réel/virtuel, $\mathrm{n}^{\circ} 2$, [ version électronique ] http://reelvirtuel.univparis1.fr/index.php?/revue-en-ligne/a-coutant---s-thomas/2/, le 16 décembre 2011.

Denouël J. et Granjon F. (sous la direction de.) 2011. Communiquer à l'ère numérique. Regards croisés sur la sociologie des usages, Presses des Mines.

Domenget J-C. 2012. De l'impératif de visibilité aux enjeux éthiques : usages de Twitter par des professionnels du web, in Proulx S., Millette M. et Heaton L. (sous la direction de.) Usages des médias sociaux : enjeux éthiques et politiques, PUQ (à paraitre).

Flichy P. 2001. L'imaginaire d'internet, La Découverte.

George E. et Granjon F. (sous la direction de.) 2008. Critiques de la société de l'information, L'Harmattan.

Giffard A. 2005. Ethique de l'internet: désirs, normes, pratiques [ version électronique ] http://alaingiffard.blogs.com/culture/2005/12/ethique_de_lint_1.html, le 16 décembre 2011.

Granjon F. 2004. De quelques éléments programmatiques pour une sociologie critique des usages sociaux des TIC, Journée d'étude les rapports société-technique du point de vue des sciences de l'homme et de la société, pp. 1-6.

Honnet A. 2004. Visibilité et invisibilité, Revue du Mauss, n²3, pp. 136-150.

Hoog E. 2009. Mémoire, Année zéro, Seuil.

Ito M. (sous la direction de.) 2008. Living and learning with new media: summary of findings from the digital youth project, Berkeley, [ version électronique ] http://digitalyouth.ischool.berkeley.edu/files/report/digitalyouth-WhitePaper.pdf, le 16 décembre 2011.

Jauréguiberry F. 2008. Sociologie des usages des technologies de la communication. "L'école française » des années 80, Actes du 18ème congrès des sociologues de langue française, 7-11 juillet, pp. 12-18.

Jouët J. 2000. Retour critique sur la sociologie des usages, Réseaux, nº 100, pp. 486-521.

Kaufmann J-C. 2006. L'entretien compréhensif, Armand Colin. 
Kline S., Dyer-Withford N. et De Peuter G. 2003. Digital Play - The interaction of Technology Culture and Marketing, McGill-Queen's University Press.

Lewis K., Kaufman J, Gonzalez M., Wimmer A. Et Kristakis N. 2008. Tastes, ties, and time: A new social network dataset using Facebook.com, Social Networks, $n^{\circ}$ 30, pp. 330-342.

Merzeau L. (sous la direction de.) 2010. Présence numérique : de la gestion d'une identité à l'exercice d'une liberté, Revue DocSI, vol. 47, n²1, pp. 28-69.

Notley T. 2009. Young People, Online Networks, and Social Inclusion, Journal of ComputerMediated Communication, $\mathrm{n}^{\circ} 14$, pp. 1208-1227.

O'Reilly T. 2005. What is web 2.0 ? [ version électronique ] http://www.oreillynet.com/pub/a/oreilly/tim/news/2005/09/30/what-is-web-20.html, le 16 décembre 2011.

Perriault J. 2009. Traces (numériques) personnelles, incertitude et lien social, Hermès, $\mathrm{n}^{\circ}$ 53, pp. 1320.

Proulx S. 2001. Usages des technologies d'information et de communication : reconsidérer le champ d'étude », Actes du 12ème Congrès de la Société Française des Sciences de l'Information et de la Communication, 10-13 janvier, Paris (France), pp. 57-66.

Proulx S. 2005. Penser les usages des technologies de l'information et de la communication aujourd'hui : enjeux - modèles - tendances, in Viera L. et Pinède N. (éditeurs.) Enjeux et usages des TIC : aspects sociaux et culturels, tome 1, PUB, pp. 7-20.

Rebillard F. 2007. Le web 2.0 en perspective. Une analyse socio-économique de l'internet, L'Harmattan.

Rebillard F. 2011. Du web 2.0 au web $^{2}$ : fortunes et infortunes des discours d'accompagnement des réseaux socionumériques, Hermès, $\mathrm{n}^{\circ}$ 59, p. 25-30.

Roqueplo P. 1983. Penser la technique : pour une démocratie concrète, Seuil.

Stenger T. 2011. La prescription ordinaire sur les réseaux socionumériques : double stratégie d'exploitation de la participation, Hermès, ${ }^{\circ}{ }^{\circ}$ 59, pp. 125-132.

Stenger T. et Coutant A. 2011a. Introduction, Hermès, nº 59, pp. 9-17.

Stenger T. et Coutant A. 2011b. Un monde d'amis ? Une ébauche de typologie sur les réseaux socionumériques, in Proulx S. et Klein A. (sous la direction de.), Connexions : communication numérique et lien social, PUN.

Vitalis A. (sous la direction de.) 1994. Médias et nouvelles technologies - Pour une socio-politique des usages, Éditions Apogée.

Voirol O. 2005. Visibilité et invisibilité : une introduction, Réseaux, n²129-130, pp. 9-36. 